\title{
Capacidad de los médicos para detectar violencia contra la mujer en la consulta externa
}

\author{
Capacity of physicians to detect violence against women in the external office
}

Francisco Javier Macías Ayala,* Osvaldo García Torres, ${ }^{\ddagger}$ Leticia Vázquez Arguelles, ${ }^{\S}$ Andrea Socorro Álvarez Villaseñor

\section{RESUMEN}

Introducción: La violencia en contra de la pareja íntima se ha documentado en todos los países del mundo. La OMS estima que mundialmente alrededor de una de cada tres mujeres ha sufrido violencia, ya sea de tipo física y/o sexual por su pareja íntima o por terceras personas en algún momento de su vida, por lo que es importante saber detectarlas y sobre todo hacerlo en actividades del sector salud. Objetivo: Identificar la capacidad de los médicos para detectar violencia contra la mujer en la consulta externa. Material y métodos: Estudio observacional, transversal, descriptivo. Previa autorización de los comités de investigación, ética y firma del consentimiento informado se realizó la encuesta PREMIS a médicos especialistas que otorgan consulta de diferentes especialidades, se realizó estadística descriptiva. Se respetó la autonomía de cada participante, en apego a las normas nacionales e internacionales en materia de investigación, principios de Belmont, declaración de Helsinki y Ley General de Salud en México. Resultados: De un total de 58 médicos se evaluaron 30 (51.7\%) médicos familiares y 28 (48.3\%) médicos de otras especialidades. Predominó el sexo masculino 32 (55.2\%), 41 (80.7\%) médicos no tienen ninguna formación previa en violencia contra la mujer, 39 (67.2\%) se sienten mínimamente preparados para detectarla. Conclusión: La capacidad de los médicos familiares y no familiares para detectar la violencia contra la mujer es menor a $50 \%$ en la población de profesionales de la salud encuestados.

Palabras clave: Detección violencia de género, detección violencia contra la mujer, detección violencia familiar.

\begin{abstract}
Introduction: Violence against intimate partners has been documented in all countries of the world. WHO estimates that worldwide around 1 in 3 women have suffered physical and/or sexual violence by their intimate partner or by third parties at some point in their life, so it is important to know how to detect them and above all to do so in health sector. Objective: To identify the capacity of doctors to detect violence against women in the outpatient clinic. Material and methods: Observational, crosssectional, descriptive study. With prior authorization from the research and ethics committees and the signing of the informed consent, the PREMIS survey was conducted with specialist physicians who provide consultations from different specialties, descriptive statistics were performed. The autonomy of each participant was respected, in adherence to national and international regulations on research, Belmont principles, the declaration of Helsinki and the general health law in Mexico. Results: Of a total of 58 doctors, 30 (51.7\%) family doctors and 28 (48.3\%) doctors from other specialties were evaluated. male sex predominated 32 (55.2\%). 41 (80.7\%) doctors do not have any previous training in violence against women, 39 (67.2\%) feel minimally prepared to detect it. Conclusion: The capacity of family and non-family doctors to detect violence against women is less than $50 \%$ in the population of health professionals surveyed.
\end{abstract}

Keywords: Gender violence detection, violence against women detection, family violence detection.

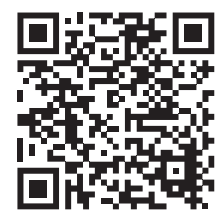

* Médico Residente de tercer año de la Especialidad de Medicina Familiar. Hospital General de Zona + Medicina Familiar No. 1. La Paz, Baja California Sur.

₹ Médico Especialista en Medicina Familiar. Profesor Titular de la Residencia en Medicina Familiar. Hospital General de Zona + Medicina Familiar No. 1. La Paz, Baja California Sur.

${ }^{5}$ Médico Especialista en Ginecología y Obstetricia. Hospital General de Zona + Medicina Familiar No. 1. La Paz, Baja California Sur. " Doctora en Ciencias. Coordinadora Auxiliar de Investigación. Delegación Regional en Baja California Sur del Instituto Mexicano del Seguro Social. La Paz, Baja California Sur.

Correspondencia: ASAV, andrea_surgery@icloud. com Conflicto de intereses: Ninguno.

Citar como: Macías AFJ García TO, Vázquez AL, Álvarez VAS. Capacidad de los médicos para detectar violencia contra la mujer en la consulta externa. Rev CONAMED. 2021; 26(1): 27-34. https://dx.doi. org/10.35366/99125

Financiamiento: No se cuenta con financiamiento externo.

Recibido: 25/01/2021. Aceptado: 01/03/2021. 


\section{INTRODUCCIÓN}

La violencia en contra de la pareja íntima se ha documentado en todos los países del mundo, todas las culturas y todos los niveles socioeconómicos, existiendo poblaciones con más riesgo que otras. Existen, además de las agresiones de tipo físicas tales como golpes o patadas, la violencia en relaciones sexuales forzadas y otras formas de coacción sexual, maltrato psicológico, intimidación, humillación, comportamientos manipuladores, restricción de una persona a su asistencia e información, y aunque puede darse en ambos sexos, la violencia de pareja es soportada en proporción abrumadoramente mayor por las mujeres e infringida por los hombres.' Este tipo de violencia constituye un problema de salud pública y una violación de los derechos humanos de las mujeres. La OMS estima que alrededor de una de cada tres mujeres (35\%) en el mundo han sufrido violencia ya sea de tipo física y/o sexual por su pareja íntima o por terceras personas en algún momento de su vida y puede afectar a la salud física, mental, sexual y reproductiva de las mujeresy aumentar el riesgo de contraer VIH entre otras enfermedades. ${ }^{2}$ Existen datos que demuestran que las intervenciones que promueven la sensibilización y emancipación de la mujer, la prestación de orientación psicológica y las visitas domiciliarias pueden favorecer la prevención y/o reducir la violencia que ejerce la pareja en contra de la mujer, estando el médico de primer y segundo nivel en una posición privilegiada para detectar y dar seguimiento a estos casos. ${ }^{2}$ La OMS, el Banco Mundial y el Fondo de Población de las Naciones Unidas (FNUAP) reconocieron la violencia como causa de muertes, enfermedad y disminución de la calidad de vida. ${ }^{3}$ Según los resultados de la Encuesta Nacional sobre la Dinámica de las Relaciones en los Hogares (ENDIREH/2016) de las mujeres mexicanas de 15 años y más, es frecuente que vivan con varios tipos de violencia simultáneamente, lo que explica que el 66.1\% han enfrentado al menos un incidente de violencia por parte de cualquier agresor, y el $43.9 \%$ de las mujeres han sufrido violencia por parte de su actual o última pareja, esposo, novio, a lo largo de su relación. 4,5 Por su parte, el INEGI en el 2019, de acuerdo con su comunicado de prensa en el día internacional de la eliminación de la violencia contra la mujer, menciona que para el estado de
Baja California Sur, 55 de cada 100 mujeres de 15 años y más han padecido de algún incidente de violencia, ya sea por su pareja o cualquier otra persona. Entre más jóvenes, la presencia de violencia es más evidente o se denuncia más, y también se observó que en Baja California Sur, se mantuvo en tasas de defunciones por homicidio menores de 4.5, hasta 2015 que llega a 6.2 y en 2017 presenta la tasa más alta de 20.8, pero en 2018 disminuyó considerablemente a 8.2. ${ }^{6}$ Por lo tanto, el papel de los profesionales de la salud es clave para la detección y denuncia de los casos, así como promoción, coordinación y para poner en marcha estrategias efectivas para abordar y tratar la violencia contra la mujer. ${ }^{6}$ En el contexto internacional se han desarrollado herramientas para la valoración de la capacidad de respuesta de los profesionales sanitarios, tal como el cuestionario PREMIS (Physician Readiness to Manage Intimate Partner Violence Survey), el cual fue creado en Estados Unidos en el año de 2002, y permite valorar el nivel de formación, conocimientos, opiniones, y percepciones sobre la calidad en el actuar de los profesionales de la salud con respecto a los casos de violencia contra la mujer. ${ }^{7}$ La mayoría de las mujeres no hablarán de la violencia de pareja a menos que se les pregunte directamente, deben aprovecharse las oportunidades para reconocer esta violencia de forma confidencial, con la paciente en privado sin presencia de acompañantes o familiares, siendo empático sin emitir juicios de valor y siendo cuidadosos en su abordaje. ${ }^{8}$ El manejo inicial incluye aceptación del abuso, validar la experiencia de la mujer, evaluar el riesgo actual, ofrecer opciones de atención complementaria, referir los casos de violencia contra las mujeres a los servicios requeridosy documentar la respuesta de la entrevista. Es importante realizar el correcto registro cuando se atiende a mujeres que viven violencia de pareja, pudiendo ser los archivos clínicos utilizados para propósitos legales. ${ }^{8}$ Al referir a las víctimas de violencia para su atención, las instituciones de salud deben trabajar con la comunidad a un nivel intersectorial para mejorar la colaboración e integración de los servicios entre sectores, incluyendo instancias de procuración de justicia, vivienda, sanitarias, agencias locales o de desarrollo social. ${ }^{8}$ Para poder cumplir con las acciones de prevención, en México existe la Norma Oficial Mexicana NOM-046-SSA2-2005; violencia familiar, sexual y contra las mujeres. Criterios para 
la prevención y atención, que a la letra dice: Las y los prestadores de servicios de salud que otorguen atención médica a las o los usuarios involucrados en situación de violencia deberán recibir periódicamente sensibilización, capacitación y actualización en la materia previamente mencionada. ${ }^{9}$ El apoyar el desarrollo saludable, respetuoso, con relaciones sanas y sin violencia tiene el potencial de reducir los casos de violencia contra la pareja íntima y de esta manera prevenir sus efectos dañinos en los individuos, familias, comunidades y sociedades. ${ }^{10}$ Por lo que el objetivo del presente trabajo es identificar la capacidad de los médicos familiares y no familiares para detectar la violencia contra la mujer.

\section{MATERIAL Y MÉTODOS}

Se realizó un estudio observacional descriptivo tipo encuesta trasversal, en 58 médicos especialistas, 30 especialistas en medicina familiar y 28 médicos de segundo nivel, de diferentes especialidades adscritos a consultorio en turno matutino y vespertino que corresponden a los horarios disponibles en consulta externa del Hospital General de Zona con Medicina Familiar No. 1 de La Paz, Baja California Sur, México, que aceptaron participar voluntariamente en el estudio. Se excluyeron siete médicos que no aceptaron participar, o encuestas incompletas, de un universo de 65 del total de la plantilla de este hospital. Una de las premisas por las que se realizó la encuesta en consulta externa, fue debido a que los médicos cuentan población asignada, Ilevan seguimiento de los padecimientos, tiene contacto con ellos más de una vez y, por lo tanto, hay empatía entre las pacientes y su

Tabla 1: Estadísticas de fiabilidad.

\begin{tabular}{lcc}
\hline Sección & $\begin{array}{c}\text { Alfa de } \\
\text { Cronbach }\end{array}$ & $\begin{array}{c}\text { Elementos } \\
\text { aplicables, } n\end{array}$ \\
\hline II. Antecedentes & 0.393 & 30 \\
III. Opiniones & $\mathbf{0 . 8 3 1}$ & 36 \\
IV. Prácticas & $\mathbf{0 . 8 9 7}$ & 47 \\
V. Conocimientos & 0.574 & 46 \\
\hline
\end{tabular}

Alfa de Cronbach: es un coeficiente que sirve para medir la fiabilidad de una escala de medida.

\begin{tabular}{llrr}
\multicolumn{4}{c}{ Tabla 2: Sección I. Perfil. } \\
\hline \multicolumn{2}{c}{ Variable } & $\mathrm{n}$ & $\%$ \\
\hline \multirow{2}{*}{ Sexo } & Masculino & 32 & 55.2 \\
& Femenino & 26 & 44.8 \\
Tipo de médico & Familiar & 30 & 51.7 \\
& No familiar & 28 & 48.3 \\
Pacientes que & De 20 a 39 & 7 & 12.1 \\
atiende por & De 40 a 59 & 14 & 24.1 \\
semana & De 60 o más & 37 & 63.8 \\
Formación en & Sí & 17 & 19.3 \\
VCl & No & 41 & 80.7 \\
\hline
\end{tabular}

$\mathrm{VCl}=$ violencia del compañero íntimo.

médico asignado, por lo tanto se pensó que sería más fácil identificar signos o datos de violencia cuando se presente.

Se utilizó el instrumento PREMIS (Physician Readiness to Manage Intimate Partner Violence Survey) que consta de 64 ítems y cinco secciones. Valora el conocimiento sobre la violencia contra la mujer e incluye: sección I perfil, sección II antecedentes, sección III opiniones, sección IV prácticas, sección $\mathrm{V}$ conocimientos. De acuerdo con la sección, este instrumento cuenta con respuestas de opción múltiple, falso y verdadero, así como escala de respuestas tipo Likert, por lo que se realizó un análisis de fiabilidad para cada una de las secciones (Tabla 7).

Al realizar el cuestionario PREMIS se procedió a recopilar la información y analizar los datos con el software de estadística SPSS V21, utilizando porcentajes y frecuencias para variables cualitativas, así como medidas de tendencia central para las variables de tipo cuantitativas. Estudio con riesgo menor al mínimo según la Ley General de Salud en México. Este estudio fue autorizado por el comité de investigación y el comité de ética en investigación de la unidad hospitalaria, obteniendo el número de registro R-2019-301-024.

\section{RESULTADOS}

Se realizó un estudio observacional sobre capacidad de los médicos de la consulta externa para detectar violencia contra la mujer en un 


\begin{tabular}{|c|c|c|c|}
\hline Ítem & Opciones & $\mathrm{n}$ & $\%$ \\
\hline $\begin{array}{l}\text { Identificar indicadores de VCI basándose en la } \\
\text { historia clínica y el examen físico }\end{array}$ & $\begin{array}{l}\text { Nada preparado } \\
\text { Mínimamente preparado } \\
\text { Algo preparado } \\
\text { Moderadamente preparado/a } \\
\text { Preparado/a } \\
\text { Bien preparado/a }\end{array}$ & $\begin{array}{r}4 \\
39 \\
6 \\
3 \\
3 \\
3\end{array}$ & $\begin{array}{r}6.9 \\
67.2 \\
10.3 \\
5.2 \\
5.2 \\
5.2\end{array}$ \\
\hline $\begin{array}{l}\text { Documentar la historia de } \mathrm{VCl} \text { y los hallazgos del } \\
\text { examen físico en la historia clínica }\end{array}$ & $\begin{array}{l}\text { Mínimamente preparado } \\
\text { Algo preparado } \\
\text { Moderadamente preparado/a } \\
\text { Preparado/a } \\
\text { Bien preparado/a } \\
\text { Muy bien preparado/a }\end{array}$ & $\begin{array}{r}44 \\
6 \\
2 \\
4 \\
1 \\
1\end{array}$ & $\begin{array}{r}75.9 \\
10.3 \\
3.4 \\
6.9 \\
1.7 \\
1.7\end{array}$ \\
\hline Realizar referencias apropiadas en casos de $\mathrm{VCl}$ & $\begin{array}{l}\text { Mínimamente preparado } \\
\text { Algo preparado } \\
\text { Moderadamente preparado/a } \\
\text { Preparado/a } \\
\text { Bien preparado/a } \\
\text { Muy bien preparado/a }\end{array}$ & $\begin{array}{r}44 \\
7 \\
3 \\
2 \\
1 \\
1\end{array}$ & $\begin{array}{r}75.9 \\
12.1 \\
5.2 \\
3.4 \\
1.7 \\
1.7\end{array}$ \\
\hline ¿Cuánto siente que sabe sobre VCI? & $\begin{array}{l}\text { Muy poco } \\
\text { Un poco } \\
\text { Moderadamente } \\
\text { Una cantidad justa } \\
\text { Bastante } \\
\text { Mucho }\end{array}$ & $\begin{array}{r}8 \\
8 \\
30 \\
7 \\
4 \\
1\end{array}$ & $\begin{array}{r}13.8 \\
13.8 \\
51.7 \\
12.1 \\
6.9 \\
1.7\end{array}$ \\
\hline
\end{tabular}

$\mathrm{VCl}=$ violencia del compañero íntimo.

total de 58 médicos del Hospital General de Zona + Medicina Familiar No. 1, contando con 30 (51.7\%) médicos familiares y 28 (48.3\%) médicos de otras especialidades. La capacidad se midió a través de la herramienta PREMIS (Physician Readiness to Manage Intimate Partner Violence Survey). Para la sección I, perfil, predominó el sexo masculino con 32 médicos (55.2\%). De la muestra total, 37 médicos atienden a 60 o más pacientes por semana, además se identificó que 41 contestaron que no tienen o han recibido ninguna formación previa en violencia contra la mujer (Tabla 2). En la sección II, antecedentes, se identificaron indicadores de violencia contra la mujer basándose en la historia clínica y el examen físico en el $67.2 \%$ de los participantes. El 75.9\% de los médicos identifican que se sienten mínimamente preparados para documentar la historia de violencia contra la mujer y los hallazgos del examen físico en la historia clínica, así como para realizar referencias apropiadas en casos de violencia contra la mujer. De acuerdo con los conocimientos previos, el 51.7\% identifica que se siente moderadamente preparado (Tabla 3). En la sección III, opiniones, el 81\% está de acuerdo para el ítem «no tengo la suficiente preparación para ayudar a las pacientes a afrontar situaciones de violencia contra la mujer»; sin embargo, sí se sienten cómodos hablando de violencia contra la mujer con sus pacientes, sí son capaces de abordar el tema aun cuando la paciente vaya por algún otro padecimiento. El $81 \%$ refiere que identifica y conoce los requerimientos legales para reportar casos sospechosos de violencia contra la mujer. 
Pero debido a la carga laboral, el 46.6\% comenta que está demasiado ocupado/a para participar en un equipo multidisciplinario para manejar casos de violencia contra la mujer (Tabla 4). En la sección IV, prácticas, ¿cuántos diagnósticos nuevos de violencia contra la mujer ha realizado en los últimos seis meses? Resalta que el 22.4\% ha reportado desde 1-5 diagnósticos nuevos.

\begin{tabular}{llrc} 
& Tabla 4: Sección III. Opiniones. & & \\
\hline Ítem & Opciones & $n$ & $\%$ \\
\hline No tengo la suficiente preparación & En desacuerdo & 9 & 15.5 \\
para ayudar a las pacientes a afrontar & De acuerdo & Totalmente de acuerdo & 81 \\
situaciones de VCl & Totalmente en desacuerdo & 47 & 3.4 \\
Me siento cómodo/a hablando de VCl con & En desacuerdo & 1 & 1.7 \\
mis pacientes & De acuerdo & 9 & 81 \\
& Totalmente de acuerdo & 47 & 1.7 \\
Conozco los requerimientos legales para & Totalmente en desacuerdo & 1 & 3.4 \\
reportar casos sospechosos de VCl & En desacuerdo & 2 & 24.1 \\
Estoy demasiado ocupada/o para & De acuerdo & 14 & 72.4 \\
participar en un equipo multidisciplinario & Totalmente en desacuerdo & 42 & 6.9 \\
para manejar casos de VCl & De acuerdo & 4 & 41.4 \\
& Totalmente de acuerdo & 24 & 5.6 \\
\hline
\end{tabular}

$\mathrm{VCl}=$ violencia del compañero íntimo.

Tabla 5: Sección IV. Prácticas.

\begin{tabular}{|c|c|c|c|}
\hline Ítem & Opciones & $\mathrm{n}$ & $\%$ \\
\hline $\begin{array}{l}\text { ¿Cuántos diagnósticos nuevos (un caso agudo } \\
\text { de VCI continuada, paciente que revela una } \\
\text { historia pasada de VCI) de VCI ha realizado en } \\
\text { los últimos seis meses? }\end{array}$ & $\begin{array}{l}\text { Ninguno } \\
\text { De } 1 \text { a } 5 \\
\text { No aplicable }\end{array}$ & $\begin{array}{r}43 \\
13 \\
2\end{array}$ & $\begin{array}{r}74.1 \\
22.4 \\
3.4\end{array}$ \\
\hline Pregunto a todas las nuevas pacientes mujeres & $\begin{array}{l}\text { Sí } \\
\text { No }\end{array}$ & $\begin{array}{r}6 \\
52\end{array}$ & $\begin{array}{r}10.3 \\
89.7\end{array}$ \\
\hline $\begin{array}{l}\text { ¿Hay un protocolo de manejo de casos de } \mathrm{VCl} \\
\text { en su servicio? }\end{array}$ & $\begin{array}{l}\text { Sí, y es ampliamente usado } \\
\text { Sí, y se usa hasta cierto punto } \\
\text { Sí, pero no se usa } \\
\text { No } \\
\text { No estoy segura(o) }\end{array}$ & $\begin{array}{r}4 \\
25 \\
4 \\
9 \\
16\end{array}$ & $\begin{array}{r}6.9 \\
43.1 \\
6.9 \\
15.5 \\
27.6\end{array}$ \\
\hline $\begin{array}{l}\text { ¿Siente que tiene conocimiento adecuado } \\
\text { sobre recursos para referencia de pacientes con } \\
\text { VCl en su comunidad? }\end{array}$ & $\begin{array}{l}\text { Sí } \\
\text { No } \\
\text { No estoy segura } \\
\text { No estoy haciendo práctica } \\
\text { clínica }\end{array}$ & $\begin{array}{r}22 \\
11 \\
24 \\
1\end{array}$ & $\begin{array}{l}37.9 \\
19 \\
41.4 \\
1.7\end{array}$ \\
\hline
\end{tabular}

$\mathrm{VCl}=$ violencia del compañero íntimo. 
Tabla 6: Sección V. Conocimientos.

\begin{tabular}{llrl}
\hline Ítem & Opciones & $n$ & $\%$ \\
\hline ¿Cuál es el factor de riesgo más & Edad (menor de 30 años) & 4 & 6.9 \\
importante para ser víctima de VCI? & Pareja abusa de alcohol/drogas & 43.1 \\
& Cénero (ser mujer) & 25 & 37.9 \\
& Historia familiar de violencia & 22 & 46.6 \\
& No sabe/no contesta & 27 & 13.8 \\
¿Cuál de las siguientes es & Tienes problemas controlando su ira & 36.2 & 41.4 \\
generalmente cierta sobre los & Utilizan la violencia para controlar a sus & 21 & 48.3 \\
abusadores? & parejas & 28 & 15.5 \\
& Son violentos porque beben o usan drogas & 9 & 39.7 \\
¿Cuáles de los siguientes son signos & Dolor crónico sin causa aparente & 23 & 58.6 \\
de que una paciente puede haber & Ansiedad & 34 & 31 \\
sufrido violencia por su pareja? & Abuso de sustancias & 40 & 69.4 \\
& Lesiones frecuentes & 33 & 56.9 \\
\hline
\end{tabular}

$\mathrm{VCl}=$ violencia del compañero íntimo.

Para la práctica de la búsqueda intencionada de violencia, es decir, preguntar de manera dirigida sin esperar que la mujer lo mencione, casi el 90\% no pregunta. El 43.1\% de los médicos reconoce y usa el protocolo de manejo en casos de violencia (Tabla 5). En la sección V, conocimientos, para la pregunta ¿cuál es el factor de riesgo más importante para ser víctima de violencia? El 69\% refiere las lesiones frecuentes, el 46.6\% consideran que debería existir historia familiar de violencia y el 48.3\% la existencia de exceso de alcohol y drogas es el factor detonante de la violencia por parte de los abusadores (Tabla 6). Se realizó un análisis de fiabilidad del instrumento para las secciones II, III, IV y $\vee$, se encontró lo siguiente: de acuerdo con el análisis de fiabilidad por secciones, el instrumento es sensible para identificar opiniones consistentes en falta de preparación, estar demasiado ocupado, aun cuando tengan conocimientos legales en temas de violencia. Así como es sensible para identificar que en la práctica clínica no se busca de manera intencionada la presencia de violencia. Sin embargo, para antecedentes y conocimientos, existe variabilidad en la formación de los médicos familiares y no familiares en temas de violencia (Tabla 7).

\section{DISCUSIÓN}

La violencia contra la mujer, no sólo como un fenómeno individual sino como un problema social y de salud pública, toma vital importancia el rol y la actuación de los profesionales de la salud, por lo que en el presente estudio se obtuvo una tasa de respuesta del 89\% (58 médicos) comparado con otros estudios similares donde sólo se obtiene alrededor del 50\% de tasa de respuesta de los encuestados como se observa en el estudio de Díaz Vigón y colaboradores (2017), dado que el cuestionario trata de un tema de alta sensibilidad y supone autovaloración del personal de salud, algunos participantes se abstuvieron de contestar el cuestionario, también algunos manifestaron falta de tiempo." En cuanto a la identificación de al menos un caso nuevo de violencia contra la mujer en los últimos seis meses, el $74.1 \%$ de profesionales de salud no identificó casos nuevos y el $22.4 \%$ sólo de uno a cinco casos a pesar de la escasez de formación recibida en el tema, lo cual concuerda con el estudio antes mencionado en el cual ninguno de los profesionales respondió que había identificado un caso nuevo en los últimos seis meses en unidades de atención primaria, llegando a 
un máximo de $27.1 \%$ en otras unidades de atención primaria encuestadas y hasta $40 \%$ en atención médica continua; en contraste, sólo el 19.3\% de los encuestados refiere haber recibido alguna formación en temas de violencia del compañero íntimo comparado con el 78\% de los encuestados en el estudio citado." Sobre la preparación para identificar indicadores de $\mathrm{VCl}$ según historia clínica y el examen físico, los profesionales de salud encuestadosse perciben mínimamente preparados, el 67.2\%, comparado con el grupo de encuestados de Diaz Vigón y colaboradores, donde el 33.1\% se siente algo preparado/a y el 26.6\% mínimamente preparado, destacando en ambas la escasez en la autopercepción de conocimientos y formación en temas de $\mathrm{VCl}$ en los profesionales de la salud." En este estudio, el $81 \%$ de encuestados está de acuerdo con sentirse cómodo/a hablando de VCl con sus pacientes a diferencia del $35.1 \%$ habiendo mucha disparidad probablemente debido a diferencias en la comunicación o culturales en los encuestadosy su interacción con su población de pacientes, teniendo la confianza necesaria para abordar el problema o no." El 46.6\% de los encuestados estuvo de acuerdo en que está demasiado ocupado/a para participar en un equipo multidisciplinario para manejar casos de violencia del compañero íntimo comparado con el 31.1\%, siendo en ambos importante la cantidad de profesionales de la salud que consideran que el tiempo con que disponen no es suficiente para participar en el manejo de los casos de violencia, por lo que el tiempo es un factor de relevancia actuando como barrera para la disposición de los profesionales de la salud para atender a las mujeres y abordar el tema adecuadamente." El 56.9\% de los profesionales que respondieron afirma que hay un protocolo de actuación ante casos de violencia en su unidad de servicio, frente al $43.1 \%$ que refiere no conocer su existencia, de los que afirman conocerlo el 6.9\% refiere que es ampliamente usado, el $43.1 \%$ que se usa hasta cierto punto y el 6.9\% restante que no se usa a pesar de existir, siendo similar con los encuestados por Díaz Vigón y su equipo, donde se observó que el 63\% de los encuestados afirma que hay un protocolo, 37\% no conocen su existencia, de los que afirman conocerlo el $72.1 \%$ refiere que se utiliza hasta cierto punto, $16.3 \%$ que es ampliamente usado y el $11.5 \%$ que no se usa, en ambos estudios la acción más realizada por los profesionales de la salud encuestados es referir a trabajo social." Asimismo, acerca de la preparación para documentar la historia de violencia y examen físico en la historia clínica en el presente estudio, predomina la respuesta mínimamente preparados con el 75.9\%, como también en el estudio comparado con 32.9\% mínimamente preparado siendo en ambas la respuesta más frecuente, denotando que no se tienen las herramientas necesarias para documentar adecuadamente los casos de $\mathrm{VCl}$, concordando también con Morales (2015) donde se encontraron graves dificultades en lo que al registro de violencia contra la mujer en la historia clínica se refiere, y también para utilizar el código de diagnóstico adecuado provocando ambigüedades. ${ }^{12}$ Los signos sugestivos más frecuentes de que una paciente que pudieron haber sufrido violencia por su pareja según los profesionales de la salud encuestados son lesiones frecuentes, seguido de ansiedad y depresión, siendo esta última una de las patologías que se observan con mayor prevalencia en mujeres que en hombres, incluyendo alteraciones distímicas de mayor severidad psicopatológica equiparable con los resultados obtenidos en la herramienta «violencia social encubierta hacia la mujer (VISEM)» Vinagre (2019); asimismo, según los profesionales de la salud, la violencia ejercida sobre la mujer tiene relación con el consumo de alcohol o drogas por parte de su pareja, y el utilizar la violencia para controlar a la víctima, concuerda con el VISEM cuyos resultados encuentran relación entre la baja autoestima, la sumisión, la dependencia emocional a la pareja, misma que puede usar el abusador para ejercer violencia sobre su pareja y de esta manera controlarla.13 El 10.3\% de los encuestados pregunta a todas las nuevas pacientes mujeres en su consulta acerca de violencia, a diferencia de $44 \%$ de los profesionales de salud encuestados por Fordsdike, O'Connor, Castle, Hegarty (2018), el 10.5\% manifestaron que no tienen las habilidades necesarias para discutir sobre violencia con víctimas mujeres. ${ }^{14}$ El $46.6 \%$ de profesionales de salud encuestados sugiere que la historia familiar de violencia es el factor de riesgo más importante para ser víctima de violencia, concuerda con López Angulo (2011) donde los datos reflejaron que no haber presenciado o recibido violencia en su niñez se comportó como factor protector con un OR = 0.14 para no permitir que su cónyuge le infrinja 
violencia; en cambio, haber sostenido relaciones de violencia psicológica con personas significativas contribuye en tres veces la probabilidad de reeditar pautas de comportamiento en su interacción de pareja y al sentido psicológico. ${ }^{75}$ En relación con las limitaciones de este estudio, se tiene en cuenta el nivel de complejidad del cuestionario PREMIS, el cual consta de numerosos ítems, también hay que tomar en cuenta que al tratarse de un tema sensible algunos participantes contestaron lo que sintieron como respuesta correcta por temor a ser juzgados en su actuar y en sus conocimientos sobre el tema de violencia contra la mujer, siendo más de la mitad de los encuestados masculinos los que podrían causar sesgo; sin embargo, estos resultados sirven como un llamado de alerta para reforzar la capacitación, sensibilización del equipo de salud para identificar y actuar de forma oportuna ante la violencia.

\section{CONCLUSIONES}

La capacidad de los médicos adscritos a la consulta externa de diferentes especialidades para detectar la violencia contra la mujer es menor al 50\% en la población de profesionales de la salud encuestados en la unidad, tomando en cuenta las diferentes variables tales como antecedentes, acciones, percepciones y conocimiento acerca de la violencia contra la mujer, lo que pone de manifiesto la necesidad de contar con más capacitación y sensibilización acerca de estos temas y búsqueda intencionada de casos en la consulta.

\section{REFERENCIAS}

1. OPS. Informe mundial sobre la violencia y la salud. Washington, D.C.: Organización Panamericana de la Salud, Oficina Regional para las Américas de la Organización Mundial de la Salud; 2003. Disponible en: https://apps.who. int/iris/bitstream/handle/10665/43437/9275324220_spa.pdf

2. Organización Mundial de la Salud. OMS: Violencia contra la mujer. 2014. Disponible en: http://www.who.int/ mediacentre/factsheets/fs239/es/

3. Secretaría de Salud. Programa de prevención y atención de la violencia. Disponible en: http://www.salud.gob.mx/ unidades/cdi/documentos/DOCSAL7758.pdf

4. Ley general de acceso de las mujeres a una vida libre de violencia, Nueva Ley publicada en el Diario Oficial de la Federación el $7^{\circ}$ de febrero de 2007 texto vigente, última reforma publicada DOF 13-04-2018. Disponible en: http://www.diputados.gob.mx/LeyesBiblio/pdf/ LGAMVLV_130418.pdf

5. INEGI. Encuesta Nacional sobrela Dinámica de las Relaciones en los Hogares. ENDIREH. INEGI; 2017. Disponible en: https://www.inegi.org.mx/contenidos/saladeprensa/ boletines/2017/endireh/endireh2017_08.pdf

6. INEGI. Estadísticas a propósito del día internacional de la eliminación de la violencia contra la mujer (25 de noviembre). 2019. Disponible en: https://www.inegi.org.mx/ contenidos/saladeprensa/aproposito/2019/Violencia2019_ Nal.pdf

7. Vives-Cases C, Torrubian-Domínguez J, CarrascoPortiño M, Espinar-Ruiz E, Gil-González, D, Goicolea I et al. Validación de la versión española del cuestionario Physician Readiness to Manage Intimate Partner Violence Survey (PREMIS). Rev Esp Salud Pública. 2015; 89 (2): 173-190. Disponible en: http://scielo.isciii.es/scielo. php?script=sci_arttext\&pid=\$1135-57272015000200006

8. Guías de Práctica Clínica. Detección y atención de la violencia de pareja y de la violencia sexual en el primer y segundo nivel de atención. Disponible en: http://www.cenetec.salud.gob.mx/descargas/gpc/ CatalogoMaestro/294_SSA_10_Violencia_pareja_Y_sexual/ CRR_SSA_294_10.pdf

9. NOM-046-SSA2-2005. Violencia familiar, sexual y contra las mujeres. Criterios para la prevención y atención. CNDH; 2006. Disponible en: http://www.cndh.org.mx/ sites/all/doc/Programas/VIH/LeyesNormasReglamentos/ NormaOficialMexicana/NOM-046-SSA2-2005_ ViolenciaFamiliarSexual.pdf

10. Centers of Disease Control and Prevention. Intimate partner violence consequences. Available in: https:// www.cdc.gov/violenceprevention/pdf/IPV-Factsheet.pdf

11. Díaz Vigón N, Fernández Gutiérrez L, Fernández Raigada RI, Rubio Domínguez J, Santos Granda MI. Conocimientos, opiniones y creencias de los profesionales de atención primaria en relación a la violencia de género. RqR Enfermería Comunitaria. 2017; 5 (2): 35-49.

12. Morales López R. Actitudes y actuación de los médicos de familia respecto a la violencia de género en la Comunidad Autónoma de la Región de Murcia [Tesis]. España: Universidad de Murcia; 2015.

13. Vinagre González AM. Violencia social encubierta hacia la mujer y su repercusión en la salud [Tesis]. Madrid: Universidad Complutense de Madrid; 2019.

14. Forsdike K, O'Connor M, Castle D, Hegarty K. Exploring Australian psychiatrists' and psychiatric trainees' knowledge, attitudes and preparedness in responding to adults experiencing domestic violence. Australas Psychiatry. 2019; 27 (1): 64-68.

15. López Angulo LM. Violencia hacia la mujer por su pareja. Intervenciones orientadas a su manejo integral [Tesis]. Cienfuegos: Universidad de Ciencias Médicas de Cienfuegos; 2011. 